Reprod. Nutr. Dévelop., 1982, 22 (6), 1025-1034.

\title{
Effet de l'hypophysectomie sur la survie, la croissance pondérale et la consommation alimentaire du poulet
}

\section{J. GOUSSOPOULOS}

Station de Physiologie animale, I.N.R.A.

E.N.S.A., Place Viala, 34060 Montpellier Cedex.

Summary. Effect of hypophysectomy on survival, weight gain and food intake in chickens.

Hypophysectomy has been performed in 1, 20,50, 90 and 110-day-old chickens. The effect of the pituitary ablation depended on both the age and the sex of the birds. Chicks hypophysectomized on the day of birth survived no more than 5 days. In the other experimental groups, the later the operation was done, the longer the chicks survived, with some rare exceptions. Survival was generally better in females.

The weight gain was significantly slower in 50-day-old hypophysectomized chicks than in the controls. In the 90-day-old operated animals, slackening of weight gain was less pronounced; a catch-up weight gain began at 24 weeks and reached the control levels at about $\mathbf{4 5}$ weeks. These differences were still less marked in 110-day-old hypophysectomized chickens in which the catch-up weight gain was faster.

In all the hypophysectomized chicks, food intake was less than in the controls, but its utilization was better than in the controls, especially the pair-fed controls.

\section{Introduction.}

Le contrôle hypophysaire de la croissance a été étudié chez de nombreux mammifères, le degré d'inhibition de la croissance observé après hypophysectomie variant avec l'espèce. Plus rares sont les travaux concernant les oiseaux, en particulier les poulets immatures. Nalbandov et Card ont présenté, en 1943, des résultats relatifs à la croissance pondérale post-opératoire de poulets hypophysectomisés à 60 jours, mais sans qu'il y ait toujours eu contrôle histologique systématique de l'efficacité de l'opération. Sturkie, en 1965, a évoqué les effets de l'hypophysectomie sur la croissance. Leurs données ont été confirmées par King (1969) sur des poulets opérés entre 3 et 4 semaines, mais qui n'ont été suivis que pendant peu de semaines post-opératoires (3 à 4).

Dans cette étude nous nous proposons de décrire l'influence de l'hypophysectomie, réalisée à différents âges au cours de la croissance postnatale du poulet, sur le taux de survie, l'évolution de la croissance pondérale et la consommation alimentaire. 


\section{Matériel et méthodes.}

Ce travail porte sur 313 animaux de race New Hampshire (souche C11) provenant du troupeau élevé au laboratoire et dont la vitesse de croissance est nettement inférieure, de 65 p. 100 environ, à celle des souches modernes.

Les hypophysectomies sont réalisées aux âges de : 1 jour, 20 jours, 50 jours, 90 jours et 110 jours.

Les poulets devant être opérés sont mis en cage individuelle, 15 jours au moins avant l'intervention, dans une pièce climatisée $\left(24 \mathrm{C}^{\circ} \pm 1\right.$ ) et éclairée $14 \mathrm{~h}$ par jour.

Les prises de nourriture et d'eau de boisson, distribuées ad libitum, sont contrôlées individuellement chaque jour.

Des animaux témoins en nombre au moins équivalent sont soumis aux mêmes conditions à chaque série d'interventions et un certain nombre (25 mâles et 24 femelles) subit un simulacre d'opération.

Enfin, deux lots d'animaux sont soumis à un pair-feeding : dans le premier, 4 mâles et 4 femelles reçoivent quotidiennement la même quantité de nourriture qu'autant d'animaux correspondants opérés à 50 jours ; dans le second, 4 mâles et 4 femelles reçoivent dès la $17^{\mathrm{e}}$ semaine la même quantité hebdomadaire de nourriture qu'autant d'animaux correspondants opérés à 16 semaines.

Le tableau 1 indique la répartition de l'ensemble des animaux évoqués.

TABLEAU 1

Répartition des animaux

\begin{tabular}{lccccccccc}
\hline \multicolumn{2}{c}{ Age de l'opération en jours } & 1 & 20 & 50 & 90 & 110 & \multicolumn{2}{c}{ Total } \\
\hline $\begin{array}{l}\text { Nombre d'animaux } \\
\text { hypophysectomisés }\end{array}$ & $\circ$ & 21 & 57 & 35 & 28 & 23 & 164 & 313 \\
\cline { 2 - 9 } & $\circ$ & 28 & 35 & 37 & 27 & 22 & 149 & \\
\hline $\begin{array}{l}\text { Nombre d'animaux } \\
\text { pseudo-opérés }\end{array}$ & $\circ$ & 5 & 12 & 8 & - & - & 25 & 49 \\
\hline & $\circ$ & 5 & 11 & 8 & - & - & 24 & \\
\hline $\begin{array}{l}\text { Nombre d'animaux non opé- } \\
\text { rés soumis à un pair-feeding }\end{array}$ & $\circ$ & - & - & 4 & - & 4 & 8 & 16 \\
\hline
\end{tabular}

\section{Technique de l'hypophysectomie.}

Nous nous sommes inspirés de la technique de Schooley (1939) par voie parapharyngienne, réalisée sur le Pigeon, reprise par Assenmacher et Bayle (communication personnelle), et également appliquée par eux à la Caille.

L'animal, anesthésié au "Nembutal » par voie intrapéritonéale, est fixé sur le dos à un banc de contention spécial. La tête est prise dans une forme creuse et maintenue par le bec. L'hypophyse est abordée par la face ventrale. Après incision des plans cutanés, la trachée et l'œsophage sont repoussés latéralement, l'os basi-occipital est dénudé puis trépané à la fraise de dentiste. Une fois l'os 
spongieux traversé, entre les deux carotides, on découvre la face antéro-ventrale de la selle turcique qui est découpée. La capsule est incisée puis l'hypophyse est aspirée par une pipette de verre reliée à une pompe à vide. Le diamètre de cette pipette est variable selon l'âge de l'animal. Un piège est intercalé dans le circuit d'aspiration entre la pipette et la pompe, ce qui permet, avec une certaine habitude, de contrôler dès ce stade la qualité de l'intervention en vérifiant la taille et la forme de la glande prélevée.

Ces opérations sont toujours conduites sous contrôle visuel à l'aide d'une loupe opératoire.

Après avoir aspiré I'hypophyse, l'hémorragie à l'intérieur de la selle turcique est arrêtée par la compression et l'hémostase est consolidée en insérant un fragment de «Spongel " (Roussel).

Les animaux pseudo-opérés subissent exactement le même processus ; mais après l'incision de la dure-mère l'hypophyse mise à nu est laissée en place. L'hémorragie est stoppée et la cavité est obstruée avec de la cire à trépanation comme chez ceux dont I'hypophyse a été prélevée.

Les traitements post-opératoires se bornent aux soins des plaies par application locale de "Sulfamides" (Exoseptoplix) et au maintien des animaux dans une ambiance thermique stable $\left(24^{\circ} \mathrm{C} \pm 1\right)$.

Une étude histologique des selles turciques (Tetrachrome de Herlant) réalisées pour déterminer la présence de reliquats hypophysaires éventuels, a permis de vérifier l'efficacité de l'intervention.

\section{Résultats.}

\section{Survie des animaux hypophysectomisés.}

Le taux et la durée de survie sont variables d'un lot à l'autre. Les poussins opérés le jour de leur naissance meurent tous dans les cinq jours qui suivent. Pour les autres animaux, le tableau 2 présente l'ensemble des résultats.

Opérés à 20 jours les poulets survivent plus ou moins longtemps, cependant aucun mâle n'atteint l'âge de 9 semaines et 70 p. 100 meurent dans les trois semaines qui suivent l'opération. Chez les femelles le taux de survie est meilleur : 40 p. 100 seulement meurent dans les trois semaines post-opératoires, le taux de survie est encore de 8 p. 100 au bout d'un an, et l'une d'elles est morte à plus de deux ans. Chez les animaux opérés plus tardivement, les résultats demeurent dans l'ensemble plus favorables aux femelles chez lesquelles (à une exception près $=52$ semaines pour celles opérées à 110 jours) les taux de survie, à chaque stade de 1 à 52 semaines post-opératoires, sont d'autant meilleurs que les animaux sont opérés plus âgés.

En ce qui concerne les mâles, les meilleurs taux de survie, à chaque stade, s'observent également pour les animaux opérés le plus tardivement (110 jours). Cependant, pour les mâles opérés à 50 jours, les résultats à chaque stade postopératoire sont meilleurs que pour les mâles opérés à 90 jours ; ils sont, d'autre part (à une exception près), égaux ou supérieurs à ceux des femelles opérées au même âge de 50 jours, dont la durée moyenne de survie est également moindre.

D'une façon générale, la durée moyenne ainsi que la durée maximale de sur- 
TABLEAU 2

Taux et durée de survie post-opératoire des animaux hypophysectomisés

\begin{tabular}{|c|c|c|c|c|c|c|c|c|}
\hline \multirow{3}{*}{\multicolumn{2}{|c|}{$\begin{array}{l}\text { Age à l'opération } \\
\text { (j) }\end{array}$}} & \multicolumn{5}{|c|}{ Semaines post-opératoires } & \multirow{2}{*}{\multicolumn{2}{|c|}{$\begin{array}{l}\text { Durée de survie } \\
\text { post-opératoire }\end{array}$}} \\
\hline & & 1 & 3 & 8 & 20 & 52 & & \\
\hline & & & Taux & e sur & $(\%)$ & & $\begin{array}{l}\text { Moyenne } \\
\text { (sem) }\end{array}$ & Maximale \\
\hline \multirow{2}{*}{20} & or & 56 & 30 & 0 & & & 2 & 40 jours \\
\hline & \& & 73 & 60 & 16 & 8 & 8 & 11 & 2 ans 7 sem. \\
\hline \multirow{2}{*}{50} & $0^{*}$ & 86 & 77 & 50 & 31 & 22 & 29 & 2 ans 6 mois \\
\hline & $q$ & 74 & 66 & 51 & 48 & 22 & 26 & 1 an 2 mois \\
\hline \multirow{2}{*}{90} & o & 74 & 69 & 31 & 18 & 12 & 31 & 2 ans 4 mois \\
\hline & \% & 87 & 87 & 87 & 25 & 75 & 70 & 3 ans 8 mois \\
\hline \multirow{2}{*}{110} & O & 84 & 84 & 58 & 58 & 58 & 49 & 3 ans 10 mois \\
\hline & $q$ & 89 & 89 & 89 & 83 & 66 & 88 & 4 ans 6 mois \\
\hline
\end{tabular}

vie augmentent avec l'âge d'opération des animaux. Toutefois ces deux durées sont sensiblement plus longues pour les femelles que pour les mâles sauf, comme il vient d'être signalé, pour les animaux opérés à 50 jours.

\section{Croissance pondérale.}

Elle a été étudiée, pour des raisons relatives à la mortalité, sur des animaux opérés à 50 jours ou plus.

L'influence de l'hypophysectomie sur la croissance dépend, comme le taux de survie, de l'âge à l'intervention et du sexe des animaux opérés.

Lorsque l'ablation de l'hypophyse est réalisée à 50 jours, les animaux accusent, par rapport aux témoins, un retard de croissance pondérale qui va en s'accentuant (fig. 1A). A l'âge de 238 jours (34 semaines) le déficit par rapport aux témoins est de $500 \mathrm{~g}$ chez les mâles et de $205 \mathrm{~g}$ chez les femelles. A 315 jours (45 semaines) il devient plus important : $550 \mathrm{~g}$ chez les mâles et $340 \mathrm{~g}$ chez les femelles.

L'analyse statistique (test " $t$ " de Student sur les différences de deux moyennes) montre que, dès la $10^{\mathrm{e}}$ semaine chez les mâles et la $11^{\mathrm{e}}$ semaine chez les femelles, les différences par rapport aux témoins sont significatives au seuil de 5 p. 100 (partie ombrée de la figure $1 \mathrm{~A}$ ) et deviennent significatives au seuil de 0,1 p. 100 à la $12^{\mathrm{e}}$ semaine chez les mâles et à la $15^{\mathrm{e}}$ semaine chez les femelles.

Si la croissance des animaux opérés à 90 jours (fig. 1B) est d'abord ralentie, il se produit ensuite (à partir de la $24^{\mathrm{e}}$ semaine) un rattrapage progressif par rapport aux témoins. A 34 semaines le déficit n'est que de $110 \mathrm{~g}$ chez les mâles et $40 \mathrm{~g}$ chez les femelles. A 45 semaines les mâles opérés sont même légèrement 


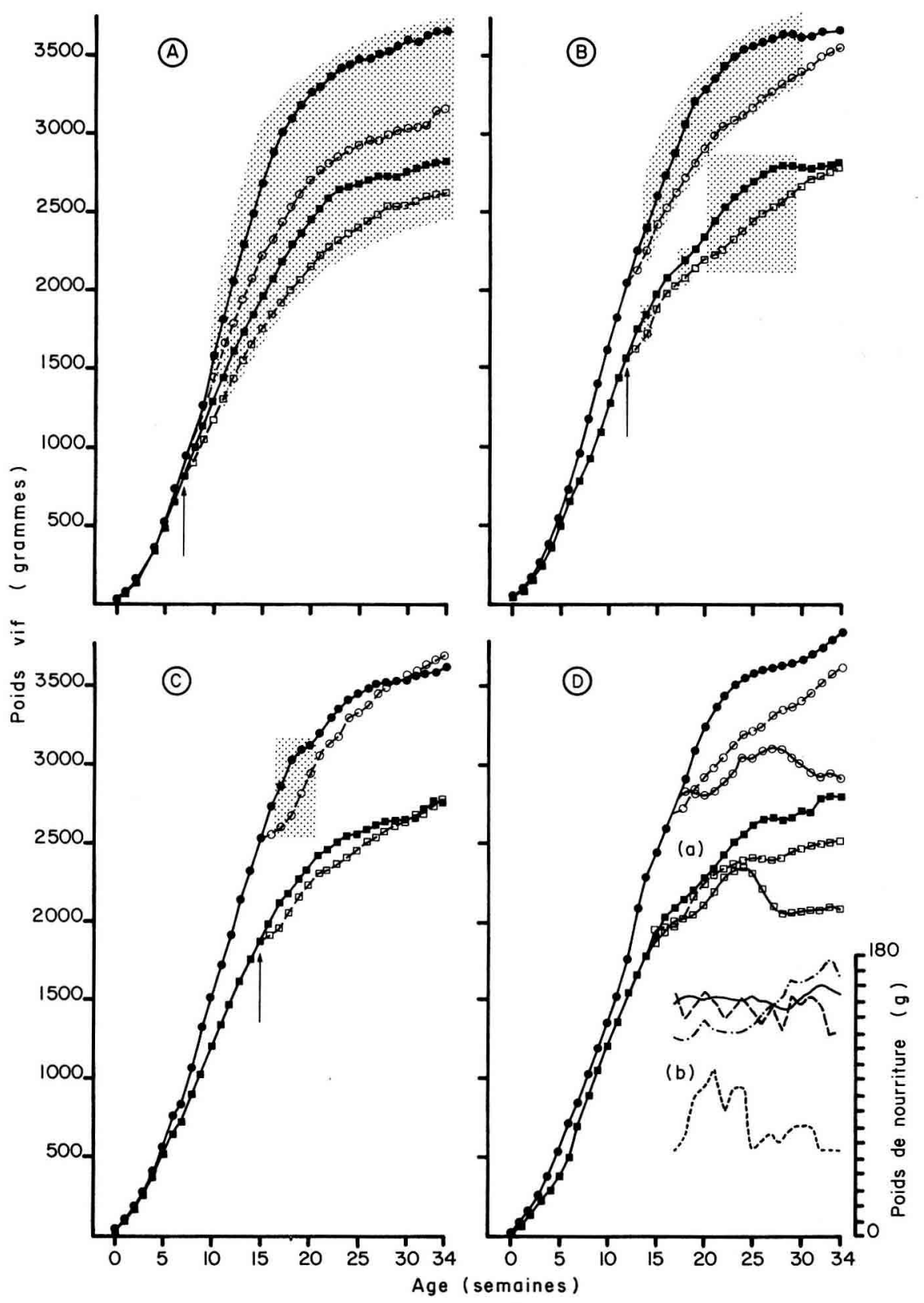

FIG. 1 A, B, C. - Courbes de croissance des animaux hypophysectomisés à 50 jours (A), à 90 jours (B), a 110 jours (C) et de leurs témoins respectifs.

-.-: mâles témoins; o----o: mâles hypophysectomisés; — : femelles témoins ; a---o: femelles hypophysectomisées.

La partie ombrée indique une différence significative au seuil de 5 p. 100 entre les moyennes des poids.

$\uparrow$ : Moment de l'hypophysectomie.

FIG. 1 D. - a) Courbes de croissance d'animaux témoins, d'animaux hypophysectomisés et d'animaux en pair-feeding par rapport à ces derniers.

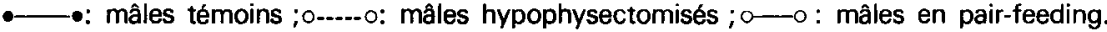

: femelles témoins ; a--.-ם: fermelles hypophysectomisées ; $0 \longrightarrow$ : femelles en pair-feeding.

b) Consommation quotidienne:

— : mâles intacts : ...... : femelles intactes ;---: mâles hypophysectomisés et en pair-feeding ;

---: femelles hypophysectomisées et en pair-feeding. 
plus lourds que les témoins ( $3890 \mathrm{~g}$ et $3800 \mathrm{~g}$ ) et les femelles ont pratiquement le même poids $(2940 \mathrm{~g}$ et $2930 \mathrm{~g})$. Statistiquement les différences observées sont plus nuancées d'un sexe à l'autre. Chez les mâles la différence est significative au seuil de 5 p. 100 de la $14^{\mathrm{e}}$ à la $30^{\mathrm{e}}$ semaine (partie ombrée) et elle atteint le seuil de 0,1 p. 100 entre la $18^{\mathrm{e}}$ et la $24^{\mathrm{e}}$ semaine. Chez les femelles, les différences ne sont significatives qu'au seuil de 5 p. 100 et seulement à la $14^{\mathrm{e}}$ semaine, à la $18^{\mathrm{e}}$ et de la $21^{\mathrm{e}}$ à la $29^{\mathrm{e}}$ semaine.

Quand l'intervention a lieu à 110 jours, ce phénomène de rattrapage est encore plus accentué chez les mâles qui, à 34 semaines, sont plus lourds que les témoins (fig. 1C), alors que les femelles opérées demeurent légèrement moins lourdes que leurs témoins. On observe ici peu de différences significatives même au seuil de 5 p. 100 sauf pour les mâles entre la $17^{\mathrm{e}}$ et la $20^{\mathrm{e}}$ semaine.

La croissance des animaux pseudo-opérés ne présente aucune différence par rapport à celle des animaux intacts.

Pour les animaux soumis à un pair-feeding les résultats sont difficilement interprétables, surtout pour ceux comparés aux hypophysectomisés à 50 jours. Dans ce dernier cas, en effet, la perte de 50 p. 100 de l'effectif en cours d'expérimentation (deux mâles et une femelle soumis au pair-feeding et un mâle hypophysectomisé) ajoutée à une importante variation individuelle et à un état cachectique des animaux rend toute l'analyse impossible.

Pour ceux comparés aux hypophysectomisés à 110 jours, les résultats sont représentés à la figure $1 \mathrm{D}$.

La consommation alimentaire de ces lots de 4 animaux est pour les mâles hypophysectomisés et les sujets en pair-feeding de $144 \mathrm{~g}$ en moyenne par jour soit 95 p. 100 par rapport à leurs témoins, et pour les femelles hypophysectomisées et en pair-feeding de $75,5 \mathrm{~g}$ par jour soit seulement 51 p. 100 par rapport à leurs témoins.

La croissance des animaux soumis au pair-feeding devient très rapidement inférieure non seulement à celle des témoins, mais également à celle des hypophysectomisés. Puis, dès la $24^{\mathrm{e}}$ semaine, chez les femelles, et la $28^{\mathrm{e}}$ semaine, chez les mâles, ces animaux amorcent une rapide perte de poids, alors que ceux sans hypophyse, dans de mêmes conditions de nourriture, de température et d'ambiance, poursuivent une croissance pondérale.

\section{Consommation journalière.}

Le tableau 3 indique les quantités journalières moyennes d'aliments consommées par les animaux hypophysectomisés et par leurs témoins, depuis l'opération jusqu'à la $20^{\mathrm{e}}$ semaine pour les animaux opérés à 50 jours et jusqu'à la $34^{\text {e }}$ semaine pour ceux opérés à 90 et 110 jours.

On note dans tous les lots une baisse de la consommation par rapport aux témoins chez les animaux hypophysectomisés. Mais, alors que chez les mâles cette baisse est d'autant plus faible que les animaux sont opérés plus tardivement, le phénomène est inversé chez les femelles : plus elles sont opérées tard, plus la baisse de consommation est importante.

La consommation journalière moyenne nettement plus faible des animaux 
TABLEAU 3

Consommation quotidienne moyenne des animaux hypophysectomisés et de leurs témoins

Mâles

Femelles

\begin{tabular}{|c|c|c|c|c|c|c|}
\hline \multirow{2}{*}{$\begin{array}{c}\text { Age } \\
\text { à } \\
\text { l'opération } \\
\text { (j) }\end{array}$} & \multicolumn{2}{|c|}{$\begin{array}{c}\text { Consommation moyenne } \\
(\mathrm{g})\end{array}$} & \multirow{2}{*}{$\begin{array}{l}\text { Consomma- } \\
\text { tion }(*)(\%)\end{array}$} & \multicolumn{2}{|c|}{$\begin{array}{l}\text { Consommation moyenne } \\
\text { (g) }\end{array}$} & \multirow{2}{*}{$\begin{array}{l}\text { Consomma- } \\
\text { tion }(*)(\%)\end{array}$} \\
\hline & $\begin{array}{l}\text { Hypophy- } \\
\text { sectomisés }\end{array}$ & Témoins & & $\begin{array}{c}\text { Hypophy- } \\
\text { sectomisés }\end{array}$ & Témoins & \\
\hline 50 & 115,1 & 139,3 & 82,6 & 92,1 & 115,4 & 79,8 \\
\hline 90 & 126,6 & 149,7 & 84,5 & 92,6 & 139,7 & 66,3 \\
\hline 110 & 145,4 & 147,8 & 98,3 & 83,4 & 146,0 & 57,1 \\
\hline
\end{tabular}

${ }^{*}{ }^{*}$ Pourcentage de consommation des hypophysectomisés par rapport aux témoins.

contrôlés à partir de 50 jours vient du fait que la période de contrôle a été plus précoce et moins étendue que celle des autres lots.

\section{Discussion.}

A notre connaissance, cette étude des effets de l'hypophysectomie est la première réalisée avec un nombre aussi important d'animaux, comportant 5 stades opératoires différents en cours de croissance, un contrôle de la consommation alimentaire post-opératoire, et un contrôle histologique systématique de l'efficacité de l'opération.

Ce dernier nous a d'ailleurs permis de constater que lorsqu'il n'y a pas de reliquats de tissu hypophysaire sur les coupes sériées de selle turcique, l'efficacité de l'intervention se traduit aussi par les particularités très prononcées qui surviennent, au bout d'un certain temps, chez les animaux et dont certaines avaient déjà été notées par Nalbandov : régression de la crête, qui devient très réduite et très pâle, aspect du plumage privé de ses couleurs vives, manque de " barbules " des plumes, et enfin comportement calme, surtout chez les mâles qui perdent leur agressivité.

\section{Survie des animaux.}

La survie des animaux opérés varie en fonction de l'âge au moment de l'intervention, ainsi qu'en fonction du sexe.

Tous les animaux opérés le jour de leur naissance meurent dans les 4 jours qui suivent, ce qui n'est pas dû à la manipulation opératoire sur des animaux fragiles, puisque les témoins ayant subi un simulacre d'opération survivent. II est difficile d'expliquer les meilleurs taux de survie obtenus pour les mâles opérés à 50 jours, par rapport à ceux opérés à 90 jours, la littérature ne fournissant pas d'exemples comparables. Nalbandov et Card (1943) signalent que les concentrations plasmatiques en acides gras libres et en glucose ne seraient pas affectées par l'hypophysectomie de poulets âgés de 61 jours. Toutefois, comme pour les femelles, la durée moyenne de survie des mâles est d'autant plus longue que l'opération a eu lieu plus tardivement. 
D'autre part, aucune cause de mortalité autre que l'hypophysectomie n'a pu être décelée. Les publications fournissant des données relatives à l'influence de I'hypophysectomie sur la survie des oiseaux sont également peu nombreuses. Ma et Nalbandov (1963) trouvent, chez des poulets en croissance, hypophysectomisés, une mortalité d'environ un tiers dans les 2 mois qui suivent l'intervention. Bayle (1968) a expérimenté sur le Pigeon, le Canard et la Caille préhypophysectomisés ayant atteint leur maturité somatique. Le taux de survie est nettement plus élevé chez le Pigeon que chez le Canard ou la Caille. Schooley (1939) a signalé aussi les conditions de survie relativement favorables du pigeon hypophysectomisé.

\section{Croissance pondérale.}

Le ralentissement de croissance observé dès après l'opération chez les animaux hypophysectomisés n'est pas dû au choc opératoire car il a été vérifié dans chaque cas que des témoins pseudo-opérés ne subissent aucun ralentissement de croissance. Nalbandov et Card (1943) ont également constaté, après hypophysectomie, un retard de croissance qui semble plus important chez les femelles (croît de 18 p. 100 chez les mâles hypophysectomisés contre 22 p. 100 chez les témoins et de 21 p. 100 chez les femelles hypophysectomisées contre 33 p. 100 chez les témoins). Par contre, ils n'ont pas enregistré de rattrapage pondéral, probablement parce que leurs animaux ont été opérés avant 90 jours et n'ont pas été conservés après 24 semaines (un rattrapage pondéral ne s'est manifesté pour nos animaux que chez ceux opérés à 90 jours ou plus, et à partir de la 24 e semaine chez les opérés à 90 jours).

Bayle (1968) constate une chute de poids chez des oiseaux hypophysectomisés, mais il s'agit dans ce cas d'animaux adultes, donc non en état de présenter des possibilités de rattrapage (chute de poids de 14 p. 100 en 5 semaines chez la Caille, 18 p. 100 en 5 semaines chez le Canard et 21 p. 100 en 15 semaines chez le Pigeon).

Chez les mammifères la réponse à l'hypophysectomie est très variable : elle n'affecte pas la croissance pondérale du rat jusqu'à un âge de 28 jours (Walker et al., 1952), du lapin jusqu'à un âge de 100 jours (Vezinhet, 1968b) ; la croissance pondérale est par la suite fortement ralentie. Chez l'Agneau (Vézinhet, 1968a) elle est freinée dès le plus jeune âge, alors que chez le Cobaye elle ne l'est pas (Mitchell et al., 1954 ; Knobil et Greep, 1959).

\section{Aspects nutritionnels.}

Le contrôle des prises d'aliments nous montre que les animaux sans hypophyse consomment une quantité d'aliments inférieure à celle ingérée par des animaux normaux ; ce fait a déjà été observé chez le Rat par Hahn et al. (1965) et chez l'Agneau par Vézinhet (1973). Ceci ne peut suffire à expliquer les différences de croissance enregistrées puisque les animaux hypophysectomisés à 90 jours rattrapent leur retard pondéral (et même au-delà pour les mâles opérés à 110 jours) tout en consommant moins d'aliment ; leur indice de consommation est d'ailleurs inférieur à celui des témoins. Cette meilleure utilisation alimentaire est confirmée en soumettant des animaux intacts à un pair-feeding. Ces derniers 
se sont caractérisés par une croissance pondérale nettement ralentie suivie d'une chute de poids et d'un état de maigreur extrême.

II faut encore observer qu'en consommant d'autant moins par rapport aux témoins qu'elles sont opérées plus tardivement, les femelles réagissent de façon inverse à celle des mâles, et donc qu'en cette matière l'influence du sexe se révèle aussi importante qu'en ce qui concerne le taux de survie.

Enfin, alors que la dénutrition des animaux en pair-feeding se caractérise par une forte diminution des réserves lipidiques, les animaux hypophysectomisés sont capables de synthétiser d'importantes réserves adipeuses.

Ces résultats sont difficilement interprétables: d'une part le poids des témoins et celui des hypophysectomisés sont différents et d'autre part les femelles témoins entrent en ponte à la $24^{\mathrm{e}}$ semaine, elles consomment alors plus d'aliment ; les femelles hypophysectomisées (qui ne pondent pas) en consomment moins, ce qui amplifie les différences observées. Les besoins de production et les besoins d'entretien ne peuvent ainsi être discernés.

Il faut donc admettre que l'aspect nutritionnel ne peut suffire à expliquer les bouleversements observés, selon le sexe, au niveau de la croissance globale, et qu'une étude de la croissance relative des principaux tissus se révèle nécessaire, plus particulièrement en ce qui concerne le tissu adipeux. Cette étude fera l'objet d'une prochaine publication.

Reçu en décembre 1981 Accepté en août 1982

Remerciements. - Nous tenons à remercier $\mathrm{M}$. Benevent pour les conseils et les critiques qu'il a bien voulu nous donner pour la rédaction de ce travail.

\section{Références}

BAYLE J. D., 1968. Importance respective des principaux niveaux de commande des régulations neuroendocriniennes chez les oiseaux. Th. Doct. Sci., Montpellier.

HAHN D. W., ISHIBASH T., TURNER C. W., 1965. Effect of hypophysectomy on feed intake in rats. Proc. Soc. exp. Biol. Med., 119, 1238-1241.

KING D. B., 1969. Effect of hypophysectomy of young cockerels, with particular reference to body growth, liver weight and liver glycogen level. Gen. comp. Endocr., 12, 242-255.

KNOBIL E., GREEP R. U., 1959. The physiology of growth hormone with particular references to its action in the rhesus monkey and the "species specificity " problem. Recent Progr. Horm. Res., 15, 1-69.

MA R. C. S., NALBANDOV A. V., 1963. Hormonal activity of the autotransplanted adenohypophysis. In A. V. NALBANDOV. Advances in neuroendocrinology, 306-311, University of Illinois Press, Urbana.

MITCHELL M. L., GUILLEMEIN R., SELYE H., 1954. The effect of somatotrophic hormone on the growth of normal and hypophysectomized guinea pigs. Endocrinology, 54, 111-114.

NALBANDOV A. V., CARD L. E., 1943. Effects of hypophysectomy of growing chicks. J. exp. Zool., 94, 387-413.

SCHOOLEY J. P., 1939. Technic for hypophysectomy of pigeon. Endocrinology, 25, 372-378.

STURKIE P. D., 1965. Avian physiology $2^{\text {nd }}$ ed. Comstock Associates, Ithaca, New York, $550-552$. 
VÉZINHET A., 1968a. Effet de l'hypophysectomie sur la croissance pondérale de l'agneau. C.R. Acad. Sci. Paris, sér. D, 266, 388-390.

VÉZINHET A., 1968b. Effet de l'hypophysectomie sur la croissance pondérale du lapin. C.R. Acad. Sci. Paris, sér. D, 266, 2348-2351.

VÉZINHET A., 1973. Influence de l'hypophysectomie et des traitements à l'hormone somatotrope bovine sur la croissance relative de l'agneau. Ann. Biol. anim. Bioch. Biophys., 13, 51-73.

WALKER D. G., ASLING C. W., SIMPSON M. E., LI C. H., EVANS H. M., 1952. Structural alterations in rats hypophysectomized at six days of age and their correction with growth hormone. Anat. Rec., 114, 19-47. 